\title{
Uncertainty quantification models for micro-scale squeeze-film damping
}

\author{
Xiaohui $\mathrm{Guo}^{1}, \mathrm{Jia}^{2}{ }^{2}$, Dongbin $\mathrm{Xiu}^{2}$ and Alina Alexeenko ${ }^{1, *, \dagger}$ \\ ${ }^{1}$ School of Aeronautics and Astronautics, Purdue University, West Lafayette, IN 47907, U.S.A. \\ ${ }^{2}$ Department of Mathematics, Purdue University, West Lafayette, IN 47907, U.S.A.
}

\begin{abstract}
SUMMARY
Two squeeze-film gas damping models are proposed to quantify uncertainties associated with the gap size and the ambient pressure. Modeling of gas damping has become a subject of increased interest in recent years due to its importance in micro-electro-mechanical systems (MEMS). In addition to the need for gas damping models for design of MEMS with movable micro-structures, knowledge of parameter dependence in gas damping contributes to the understanding of device-level reliability. In this work, two damping models quantifying the uncertainty in parameters are generated based on rarefied flow simulations. One is a generalized polynomial chaos (gPC) model, which is a general strategy for uncertainty quantification, and the other is a compact model developed specifically for this problem in an early work. Convergence and statistical analysis have been conducted to verify both models. By taking the gap size and ambient pressure as random fields with known probability distribution functions (PDF), the output PDF for the damping coefficient can be obtained. The first four central moments are used in comparisons of the resulting non-parametric distributions. A good agreement has been found, within $1 \%$, for the relative difference for damping coefficient mean values. In study of geometric uncertainty, it is found that the average damping coefficient can deviate up to $13 \%$ from the damping coefficient corresponding to the average gap size. The difference is significant at the nonlinear region where the flow is in slip or transitional rarefied regimes. Copyright (C) 2010 John Wiley \& Sons, Ltd.
\end{abstract}

Received 17 March 2009; Revised 17 April 2010; Accepted 21 April 2010

KEY WORDS: uncertainty quantification; squeeze-film damping; gPC expansion; rarefied flow

\section{INTRODUCTION}

Gas damping in micro-electro-mechanical systems (MEMS) has attracted a lot of interest in the past two decades [1-6]. Better understanding of gas damping effects on micro-structure dynamics

\footnotetext{
*Correspondence to: Alina Alexeenko, School of Aeronautics and Astronautics, Purdue University, West Lafayette, IN 47907, U.S.A.

†E-mail: alexeenk@purdue.edu

Contract/grant sponsor: NNSA Center for Prediction of Reliability, Integrity and Survivability of Microsystems (PRISM) at Purdue University; contract/grant number: DE-FC52-08NA28617

Copyright (C) 2010 John Wiley \& Sons, Ltd.
} 
is required for design of versatile and reliable micro-devices [7, 8]. In applications such as resonant sensors, MEMS switches and scanning probes, planar squeeze-film damping (SFD) is one of the dominant damping sources $[9,10]$. The SFD force arises when the fluid is pulled in or pushed out of a thin gap between movable structures. Micro-scale gas damping often involves non-continuum fluid flow effects due to the small gap size that can be on the same order or less than the mean-freepath of gas molecules, especially at low operating pressures that are typical for vacuum-packaged MEMS [11].

MEMS devices have been extensively used in automotive and communication applications such as micro-actuators, micro-gyroscopes and micro-accelerometers. However, in areas where high reliability is required, very few MEMS technologies have been adopted so far. For example, in aerospace industry, both the price barrier and the no-mistake rule have prevented broader applications of MEMS [12]. The bottleneck for improving MEMS reliability lies mostly in the lack of physical knowledge and a high degree of uncertainty in fabrication at the micro-scale. Hence, further research in these two areas has a potential for high societal impact.

Analysis of effects of uncertainty on device operation bridges the device physics and fabrication techniques. The reliability of MEMS device, which is understood as the probability to perform the required functions over its design lifetime, is affected mainly by two sources of uncertainty [13]. The first one is epistemic uncertainty related to the lack of understanding of fundamental failure modes and related physical models. The second one is aleatory uncertainty in parameters either due to variability in fabrication or in operating environment. The main goal of reliability analysis is to predict the stochastic behavior of the system due to these uncertainties. The dynamic behavior of MEMS typically involves a coupling between electrostatics, structural response and fluid dynamics. It is important, therefore, to quantify the effects of uncertainty in all of the coupled physical domains. A general approach for geometric uncertainty quantification in electrostatics of MEMS has been studied in References [14-16]. The work presented in this paper is focused on uncertainties in fluidic response.

For many MEMS devices, non-continuum effects of fluid flows due to small gap sizes are important. Flow non-continuity can be quantified using the Knudsen number, $K n$, which is defined as the ratio of molecular mean-free-path to the problem characteristic length. For $K n>0.01$, continuum assumptions break down and the non-continuum, or rarefied, effects must be taken into account. For small deviations from the continuum, i.e. in the so-called 'slip flow' with $0.01<K n<0.1$, the continuum Navier-Stokes model still applies, though the no-slip boundary condition needs to be replaced by a slip equation. At a higher rarefaction, the modeling has to be based on the Boltzmann equation of gas kinetic theory. Although the statistical direct simulation Monte Carlo (DSMC) [17] method for the solution of the Boltzmann equation is valid through all Knudsen regimes, it is computationally expensive for low-speed flows. A deterministic solution [18] of the Boltzmann kinetic equation is often a more suitable numerical approach for micro-device gas damping simulations and is applied here.

In this work, two models are proposed and compared. One is the generalized polynomial chaos (gPC) model [19] and the other is a compact damping model [20]. With gPC, an extension of the classical polynomial chaos (PC) developed by Ghanem [21-23], stochastic quantities are expressed as orthogonal polynomials of the input random parameters, and different types of orthogonal polynomials can be chosen to achieve better convergence [24]. While most earlier numerical work employed the Galerkin approach, collocation method has become popular since [25], which allows one to use repetitive runs of existing deterministic codes. Here, we use the pseudo-spectral gPC collocation method [26] in the damping model. As a general strategy for uncertainty quantification, 


\section{UNCERTAINTY QUANTIFICATION MODELS}

gPC can be highly accurate and efficient. On the other hand, the compact model from [20] is developed specifically for this particular SFD problem. It is a simple closed-form model consisting of a few physical parameters that are fitted based on simulations for varying Knudsen numbers and geometries. In practice, the compact model is very versatile and easy to use. In this paper, we will conduct extensive tests to verify the effectiveness of both models and then use them as predictive tools to study the effects of uncertainty in micro-scale gas damping.

The remainder of the paper is organized as follows. In Section 2, details of damping simulations and two uncertainty quantification models are described. Then, model validation and verification are presented in Section 3. Finally, in Section 4, effects of geometry and pressure uncertainties for SFD problems are analyzed and discussed. Conclusions are given in Section 5.

\section{MODELS AND METHODS}

Details of damping simulations as well as two uncertainty quantification methods are presented in this section.

\subsection{Damping model based on rarefied flow simulations}

Here, we describe the SFD problem and the rarefied flow simulation approach. Figure 1 shows the schematic of rarefied gas flows around a moving micro-cantilever. When the length, $l$, is much larger than the other two dimensions, the flow problem is assumed to be two-dimensional. Further, if the beam thickness, $t$, is much smaller than the width, $b$, sensitive geometric parameters reduce to the beam width and the gap size only.

For a micro-oscillating cantilever system, the damping ratio, $\zeta$, and the quality factor, $Q$, of its $n$th vibration mode can be defined as follows [27]:

$$
\begin{aligned}
& \zeta_{n}=\frac{c_{f}}{2 \rho_{s} b t \omega_{n}}=\frac{1}{2 Q_{n}}, \\
& \omega_{n}=\gamma_{n}^{2} \sqrt{\frac{E I}{\rho_{s} b t l^{4}}},
\end{aligned}
$$
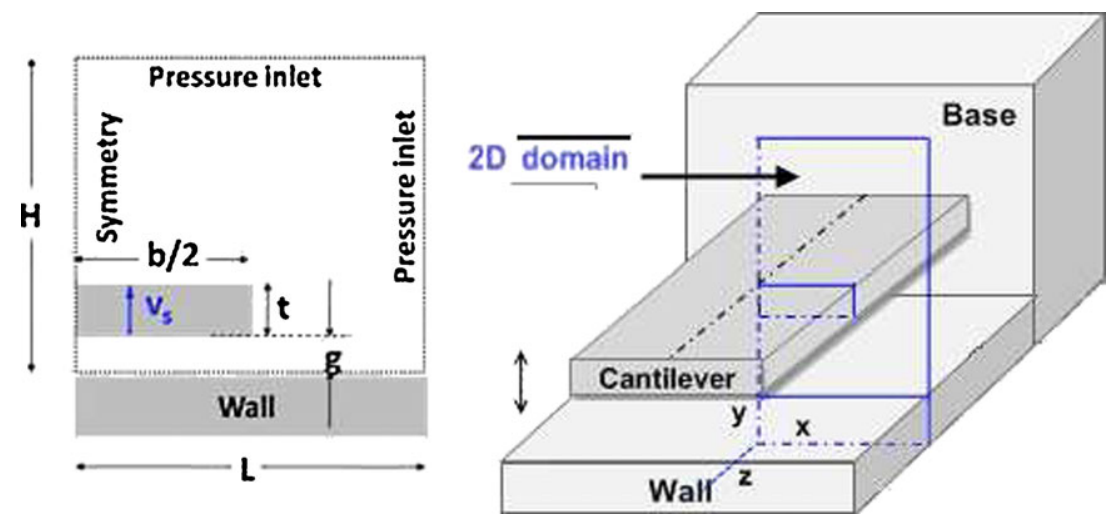

Figure 1. Schematic of the simulations for squeeze-film damping of a cantilever beam. 
where $c_{f}=F /\left(v_{s} l\right)$ is the damping coefficient, $F / l$ is the total damping force per unit length, $v_{s}$ is the speed of the cantilever motion, $I=b t^{3} / 12$ is the area moment of inertia of the beam, $E$ is the structural Young's modulus and $\rho_{s}$ is the structural density. The first three resonant frequencies correspond to $\gamma_{n}$ being $1.8751,4.6941$ and 7.8548 .

For a low-amplitude beam motion, the two-dimensional damping problem can be simulated by the quasi-steady ellipsoidal statistical Bhatnagar-Gross-Krook (ES-BGK) model assuming moderate vibration frequencies. The governing equation with respect to the velocity distribution function, $f=f(x, y, u, v)$, is given below

$$
u \frac{\partial f}{\partial x}+v \frac{\partial f}{\partial y}=v\left(f_{0}-f\right),
$$

where $u$ and $v$ are molecular velocities in Cartesian coordinates $(x, y), v$ is the intermolecular collision frequency and $f_{0}$ is the equilibrium distribution function. In ES-BGK model, $f_{0}$ is assumed to be an anisotropic Gaussian distribution in the form [28],

$$
\begin{aligned}
& f_{0}=\frac{n}{\sqrt{(2 \pi)^{3} \operatorname{det}\left[\lambda_{i j}\right]}} \cdot \exp \left(-\varepsilon_{i j} v_{i} v_{j}\right), \\
& \lambda_{i j}=\frac{1}{\operatorname{Pr}} \cdot R T \delta_{i j}+\left(1-\frac{1}{\operatorname{Pr}}\right) \cdot p_{i j} \rho, \quad \varepsilon=[\lambda]^{-1}
\end{aligned}
$$

where $p_{i j}$ is the pressure tensor, $\delta_{i j}$ is the Kronecker delta and $\mathrm{Pr}$ is the Prandtl number.

The governing equation is discretized using Cartesian coordinates in the physical space and polar coordinates in the velocity space. The discrete-velocity problem is solved using the finitevolume method with the second-order upwinding scheme, which utilizes the quadrant-splitting in the velocity space. Macroscopic parameters are calculated as moments of the velocity distribution function. The damping coefficient $c_{f}$ is obtained by integrating the computed normal pressure component along the beam cross-section and normalizing by the beam velocity $v_{s}$. Grid convergence study showed that numerical errors are within $1 \%$ for the total damping force at the considered pressure and geometry conditions.

\subsection{Uncertainty quantification models}

To quantify the uncertainty in the SFD model, the first four moments: mean, standard deviation, skewness and kurtosis [29], are used to describe the non-parametric distribution, which is usually encountered in the SFD problem. In addition, statistical tests are compared between the gPC model and the compact model.

According to parameter sensitivity study in damping simulations, it is found that the damping coefficient mainly depends on the gap size, $g$, the beam width, $b$, and the ambient pressure, $p_{A}$. Therefore, two non-dimensional variables are sufficient to describe the physics. Definitions are given below,

$$
x_{1}=\frac{b}{g}, \quad x_{2}=\frac{\lambda\left(p_{A}\right)}{b},
$$

where $\lambda\left(p_{A}\right)$ is the molecular mean-free-path at pressure $p_{A}$. 


\section{UNCERTAINTY QUANTIFICATION MODELS}

2.2.1. gPC model. The recently developed numerical methods for stochastic computations based on gPC methodology $[19,26]$ give us a way to analyze the SFD model. In this section, we will briefly introduce the gPC. An extensive review can be found in [24].

The $\mathrm{gPC}$ expansion seeks to approximate a random function via orthogonal polynomials of random variables. The $P$ th-order $\mathrm{gPC}$ approximation of any random function $u(z)$ can be obtained by

$$
u(z) \approx u_{n_{z}}^{P}(z)=\sum_{i=1}^{M} \hat{u}_{i} \Phi_{i}(z), \quad M=\left(\begin{array}{c}
n_{z}+P \\
n_{z}
\end{array}\right),
$$

where $z \in \mathbb{R}^{n_{z}}$ is a random variable, $\left\{\Phi_{i}(z)\right\}$ are the $n_{z}$-variate orthonormal polynomials that are constructed as products of a sequence of univariate polynomials in each directions of $z_{i}, i=$ $1, \ldots, n_{z}$, i.e.

$$
\Phi_{\ell}(z)=\phi_{\ell_{1}}\left(z_{1}\right) \cdots \phi_{\ell_{n_{z}}}\left(z_{n_{z}}\right), \quad \ell_{1}+\cdots+\ell_{n_{z}} \leqslant P
$$

and satisfy

$$
\mathbb{E}\left[\Phi_{j}(z) \Phi_{k}(z)\right]=\int \Phi_{j}(z) \Phi_{k}(z) p(z) \mathrm{d} z=\delta_{j k},
$$

for all $1 \leqslant j, k \leqslant M$, and $\mathbb{E}$ is the expectation operator. The Fourier coefficients $\left\{\hat{u}_{i}\right\}$ are defined as

$$
\hat{u}_{i}=\int u(z) \Phi_{i}(z) p(z) \mathrm{d} z=\mathbb{E}\left[u(z) \Phi_{i}(z)\right], \quad 1 \leqslant i \leqslant M .
$$

and can be approximated by

$$
\hat{u}_{i} \approx \sum_{j=1}^{Q} u\left(z^{(j)}\right) \Phi_{i}\left(z^{(j)}\right) w^{(j)}, \quad 1 \leqslant i \leqslant M,
$$

where $\left\{z^{(j)}, w^{(j)}\right\}_{j=1}^{Q}$ are a set of nodes and weights of cubature rule, and $u\left(z^{(j)}\right)$ is the deterministic value of $u(z)$ with fixed $z^{(j)}$.

In the SFD model, the scales of the random variables $x_{2}$ and $c_{f}$ vary from $10^{-3}$ to 10 . So to improve the accuracy, the gPC expansion is constructed for the logarithm of $c_{f}$ using $x_{1}$ and the logarithm of $x_{2}$. In reality, $x_{1}$ and $x_{2}$ are always in bounded intervals. Therefore, Legendre Polynomials are chosen as bases. Finally, considering $z=\left(x_{1}, \log x_{2}\right)$ in Equation (7), the gPC model can be described as follows:

$$
\log \left(c_{f}\right)\left(x_{1}, x_{2}\right)=\sum_{i=1}^{M} \hat{c}_{i} \Phi_{i}\left(x_{1}, \log \left(x_{2}\right)\right),
$$

where $\left\{\Phi_{i}\right\}$ are the Legendre Polynomial bases and $\hat{c}_{i}$ 's can be obtained by (11).

2.2.2. Compact gas damping model. For damping flow in slip regime $(0.001<K n<0.1)$, analytical solutions can be obtained from Reynolds equation with slip and trivial pressure boundary conditions [3]. As the Knudsen number increases, non-trivial pressure need to be taken into account. One approach for predicting the damping coefficient with low frequency damping modes gives:

$$
c_{f}=\frac{F_{0}}{v_{s} L}=\frac{(b / g)^{3}}{Q_{p r}} \cdot t
$$




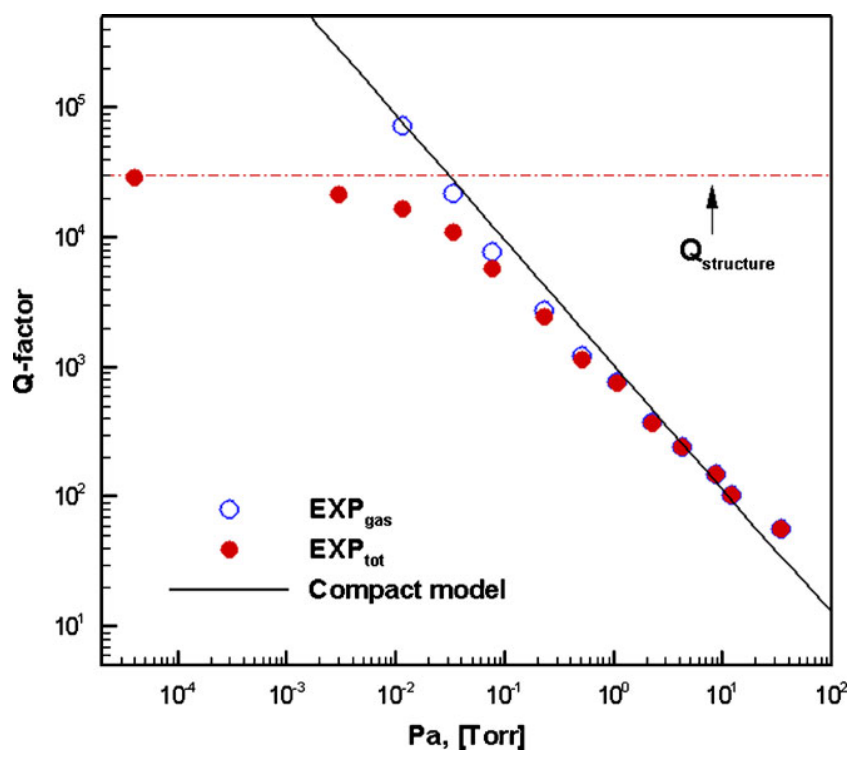

Figure 2. Comparisons of predictions by the ESBGK-based compact model and the experimental data,

Mode-1. $L=200 \mu \mathrm{m}, b=40 \mu \mathrm{m}, g=1.1 \mu \mathrm{m}, t=1.8 \mu \mathrm{m}, \rho_{s}=2.33 e+3 \mathrm{~kg} \mathrm{~m}^{-3}, E=155 \mathrm{GPa}$ [1].

where $Q_{p r}$ is the relative flow rate coefficient. At low pressures, $Q_{p r}=1+9.638\left[K n^{(g)}\right]^{1.159}$ is assumed. By substituting $Q_{p r}, x_{1}$ and $x_{2}$, Equation (13) can be rewritten as:

$$
c_{f}=\frac{x_{1}^{3}}{1+9.638 x_{1}^{1.159} x_{2}^{1.159}} \cdot t
$$

Using an expression of the form similar to that of Equation (14), a compact model based on 50 rarefied flow solutions of Equation (3)-(5) has been obtained [20] as,

$$
c_{f}=\frac{A x_{1}^{c}}{1+B \cdot x_{1}^{d} x_{2}^{e}} \cdot t
$$

where coefficients $A=10.39, B=1.374, c=3.100, d=1.825$ and $e=0.966$.

Model predictions have been compared with the experimental measurements by Zook [1] (as shown in Figure 2). The gas damping, which is obtained by subtracting the structural damping from the total measurements, agrees well with model predictions. Details about this compact damping model can be found in Reference [20].

Since the function is explicitly defined in Equation (15), expression for the output probability distribution functions (PDFs) of damping coefficient can be evaluated by the change of variables,

$$
\begin{aligned}
p\left[c_{f}\left(x_{1}\right)\right] & =p\left(x_{1}\right) \cdot\left|J_{x_{1}, c f}\right|=\left[p(g) \cdot\left|J_{g, x_{1}}\right|\right] \cdot\left|J_{x_{1}, c f}\right| \\
& =p(g) \cdot \frac{g^{2}}{b} \cdot\left|J_{x_{1}, c f}\right|
\end{aligned}
$$




$$
\begin{aligned}
p\left[c_{f}\left(x_{2}\right)\right] & =p\left(x_{2}\right) \cdot\left|J_{x_{2}, c f}\right|=\left[p\left(p_{A}\right) \cdot\left|J_{p_{A}, x_{2}}\right|\right] \cdot\left|J_{x_{2}, c f}\right| \\
& =p\left(p_{A}\right) \cdot \frac{p_{A}^{2} b}{\mu} \sqrt{\frac{2}{\pi R T}} \cdot\left|J_{x_{2}, c f}\right|
\end{aligned}
$$

where

$$
\begin{aligned}
& J_{x_{1}, c f}=\frac{\partial x_{1}}{\partial c_{f}}=\left(\frac{\partial c_{f}}{\partial x_{1}}\right)^{-1}=\left[\frac{A c \cdot x_{1}^{c-1}+(c-d) A B x_{2}^{e} \cdot x_{1}^{c+d-1}}{\left(1+B x_{1}^{d} x_{2}^{e}\right)^{2}} \cdot t\right]^{-1} \\
& J_{x_{2}, c f}=\frac{\partial x_{2}}{\partial c_{f}}=\left(\frac{\partial c_{f}}{\partial x_{2}}\right)^{-1}=\left[-\frac{A B e x_{1}^{c+d} x_{2}^{e-1}}{\left(1+B x_{1}^{d} x_{2}^{e}\right)^{2}} \cdot t\right]^{-1}
\end{aligned}
$$

\section{MODEL VERIFICATION AND VALIDATION}

In the following sections, two data sets are used for model comparison.

- $S_{1}$ : A set of two-dimensional Gauss-quadrature points generated by tensor product of onedimensional Gauss points of 4 points. The total number of points is 16 . Numerical tests verified that this is sufficient to produce accurate $\mathrm{gPC}$ expansion.

- $S_{2}$ : A set of 50 points uniformly distributed in the two-dimensional domain. Numerical tests indicated that the set is sufficient for the least-square estimation of the model parameters in the compact model (15) [20].

Both data sets cover the same ranges of non-dimensional variables, i.e. the beam aspect ratio and the gas Knudsen number, that govern the gas damping coefficient.

\subsection{Convergence study in gPC model}

Before using the gPC model, we need to determine the order of gPC expansion first. The compact model has been examined in Reference [20] for a good accuracy. Since the numerical simulation is computationally expensive to obtain, we construct the gPC expansion as shown in Equation (12) by using the estimates of compact model. More precisely, the gPC coefficients are calculated by Equation (11), in which the $c_{f}$ values are obtained by evaluating quadrature points in the compact model given by Equation (15).

The gPC expansions with different degrees have been constructed. The mean and standard deviation of the $c_{f}$ distribution from gPC are compared with those obtained by the compact model. Considering $x_{1} \in[10,18]$ and $x_{2} \in[3.6 e-3,3.6]$, for a 4th-order gPC expansion, four quadrature points are chosen from each interval so that a total of 16 points are obtained by the tensor product. The gPC coefficients are computed by the numerical simulation results on the quadrature points. In Table I, the estimates by compact model and gPC model are listed for different values of $x_{2}$. The estimates of $\mathrm{gPC}$ converge to the estimates in the compact model as the degree of gPC expansion increases. For this work, the 4th-order gPC expansion is used, which introduces relative errors less than $1 \%$. 
Table I. Convergence on degree of $\mathrm{gPC}, \Delta_{\mathrm{gPC}}=\mid \mathrm{gPC}-$ Compact $\mid /$ Compact.

\begin{tabular}{lcc}
\hline & Mean & Std \\
\hline$x_{2}=0.05$ & & \\
Compact & $7.1071 \mathrm{e}-03$ & $3.7741 \mathrm{e}-04$ \\
gPC-3 & $6.8031 \mathrm{e}-03$ & $3.8125 \mathrm{e}-04$ \\
$\Delta_{\mathrm{gPC}-3},(\%)$ & 4.2771 & 1.0166 \\
$\mathrm{gPC}-4$ & $7.1533 \mathrm{e}-03$ & $3.8160 \mathrm{e}-04$ \\
$\Delta_{\mathrm{gPC}-4},(\%)$ & 0.65 & 1.11 \\
$\mathrm{gPC}-5$ & $7.1070 \mathrm{e}-03$ & $3.7991 \mathrm{e}-04$ \\
$\Delta_{\mathrm{gPC}-5},(\%)$ & 0.0004 & 0.6614 \\
$x_{2}=0.3$ & & \\
Compact & $1.3847 \mathrm{e}-03$ & $6.5459 \mathrm{e}-05$ \\
$\mathrm{gPC}-3$ & $1.4380 \mathrm{e}-03$ & $6.7372 \mathrm{e}-05$ \\
$\Delta_{\mathrm{gPC}-3},(\%)$ & 3.8540 & 2.9228 \\
$\mathrm{gPC}-4$ & $1.3893 \mathrm{e}-03$ & $6.5551 \mathrm{e}-05$ \\
$\Delta_{\mathrm{gPC}-4},(\%)$ & 0.33 & 0.14 \\
$\mathrm{gPC}-5$ & $1.3836 \mathrm{e}-03$ & $6.5102 \mathrm{e}-05$ \\
$\Delta_{\mathrm{gPC}-5},(\%)$ & 0.0777 & 0.5454 \\
\hline
\end{tabular}

Table II. Model validation of gPC.

\begin{tabular}{lcc}
\hline Property & MSE- $S_{1}$ & Relative error $\%$ \\
\hline gPC-1 & $3.900 \mathrm{e}-3$ & 14.5 \\
gPC-2 & $6.445 \mathrm{e}-4$ & 3.76 \\
gPC-3 & $1.037 \mathrm{e}-4$ & 0.225 \\
gPC-4 & $1.603 \mathrm{e}-5$ & 0.0744 \\
\hline
\end{tabular}

\subsection{Model validation}

The purpose of model validation is to examine whether the estimates of the compact model and gPC model are consistent with the numerical simulation results. Two sets of numerical simulation results are available: the estimates on $S_{1}$ which are needed to construct gPC model, and the estimates on $S_{2}$ which are used to construct compact model.

In Table II, there is a list of the mean square error (MSE) [29] and the relative error of gPC expansion with different orders on the sixteen quadrature points. The convergence pattern on the degree of the expansion can be found clearly.

In Table III, the mean-squared errors are tabulated for gPC with degree 4 and the compact model, respectively. It can be found that both models achieve small MSE within a reasonable scale. Therefore, both models can be used for studying SFD problems.

\subsection{Model comparison}

The purpose here is to compare the output of compact model and gPC model. Both point-wise comparison and comparison of output PDFs are carried out. 
UNCERTAINTY QUANTIFICATION MODELS

Table III. Statistical tests for the gPC expansion (4th-order) and the compact model.

\begin{tabular}{lcc}
\hline Property & MSE- $S_{1}$ & MSE- $S_{2}$ \\
\hline gPC-4 & $1.603 \mathrm{e}-5$ & $2.023 \mathrm{e}-3$ \\
Compact model & $1.100 \mathrm{e}-3$ & $7.809 \mathrm{e}-4$ \\
\hline
\end{tabular}
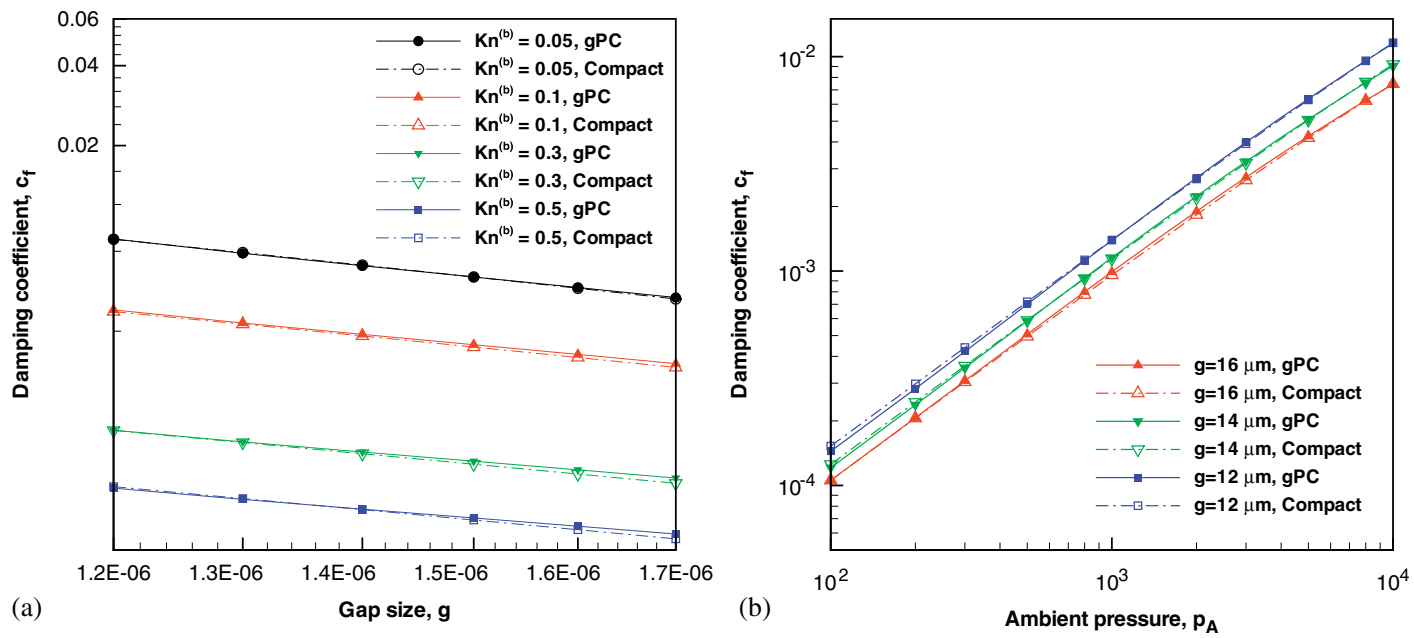

Figure 3. Comparisons of results from gPC expansion and the compact model: (a) varying gap size, unit: micron and (b) varying ambient pressure, unit: Pascal.

In Figure 3(a) and (b), damping predictions based on gPC and the compact model are compared for gap size ranging from 1.2 to $1.7 \mu \mathrm{m}$ and for ambient pressure ranging from $10^{2}$ to $10^{4} \mathrm{~Pa}$. A good agreement is observed for each pair and is consistent over all conditions of interest.

Output probability functions of the damping coefficient have been compared between gPC and compact models. Assuming the gap size and ambient pressure follows a normal distribution, moments calculated by two methods are listed in Table IV. For both cases, consider $g_{0}=1.4 \mu \mathrm{m}$, $\sigma_{g}=0.05 \mu \mathrm{m}$ and $t=1 \mu \mathrm{m}$. Except for skewness, all moments agree within $4 \%$. As expected, the compact model, which has much fewer parameters than $\mathrm{gPC}$, is not as accurate as gPC for higher moments. However, even with a 30\% relative difference in skewness, it can be found in Figure 4(a) and (b) that the distributions are very close to each other.

\section{UNCERTAINTY QUANTIFICATION FOR SFD}

Both the gPC expansion and the compact model have been validated and verified, and are capable in quantifying damping due to uncertainties in geometry and flow conditions. However, the compact model is convenient because of its simpler mathematical form than the gPC expansions in this particular problem. Therefore, we will apply the compact model in the following uncertainty quantification for SFD. 


\section{GUO ET AL.}

Table IV. Comparison of moments, $\Delta=\mid \mathrm{gPC}-$ Compact $\mid / \mathrm{gPC}$.

\begin{tabular}{lcccc}
\hline Method & Mean, $10^{-3}$ & Std, $10^{-4}$ & Skewness & Kurtosis \\
\hline$K n^{(b)}=0.05$ & & & & \\
gPC $\left(4^{\text {th }}\right)$ & 7.0741 & 3.6495 & 0.33065 & 3.2532 \\
Compact & 7.1071 & 3.7741 & 0.26441 & 3.1358 \\
$\Delta,(\%)$ & 0.466 & 3.414 & 30.033 & 3.609 \\
$K n^{(b)}=0.1$ & & & & \\
gPC $(4$ th $)$ & 3.8999 & 1.8435 & 0.31330 & 3.2311 \\
Compact & 3.8545 & 1.9143 & 0.26377 & 3.1355 \\
$\Delta,(\%)$ & 1.164 & 3.841 & 15.809 & 2.959 \\
\hline
\end{tabular}
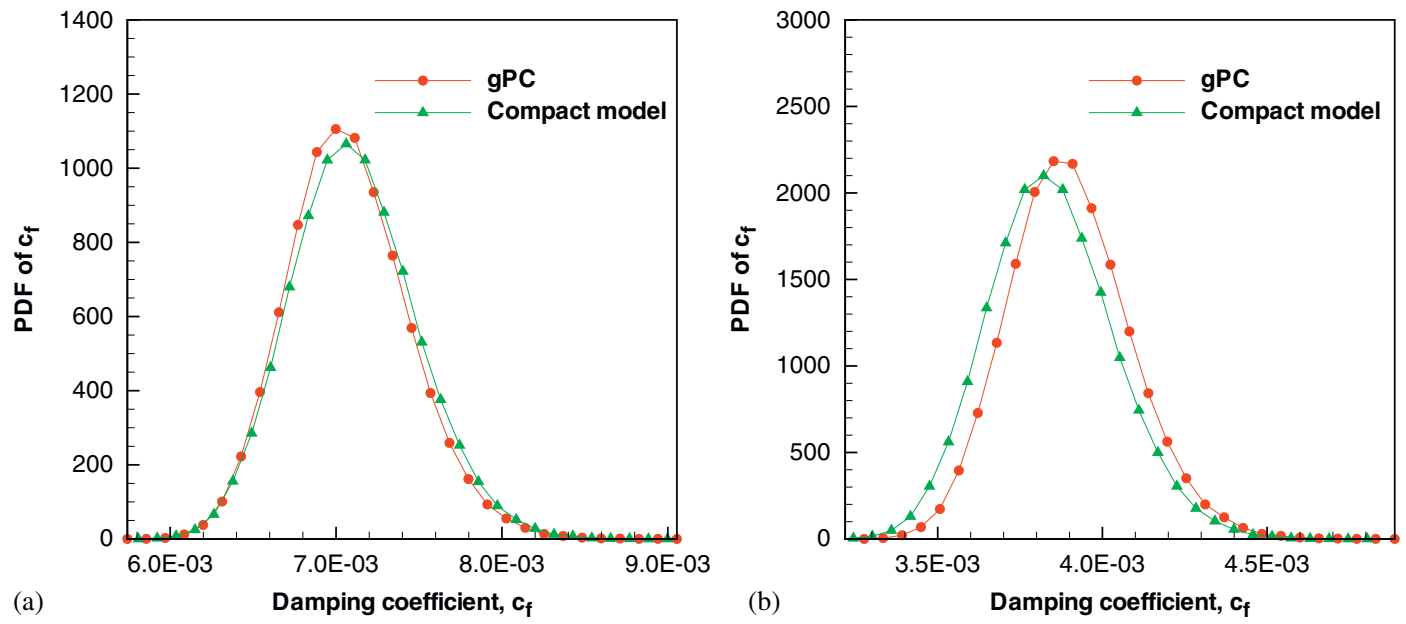

Figure 4. Comparison of the output PDF between gPC expansion and the compact model: (a) $K n^{(b)}=0.05$ and (b) $K n^{(b)}=0.1$.

\subsection{Flow fields}

The dependence of damping flow fields on the ambient pressure and the gap size can be found in Figure 5(a) and (b), respectively. The cases correspond to three gPC quadrature points with the parameters listed in Table V. From comparisons, it can be seen that the flow field produced by decreasing the ambient pressure is similar to that produced by reducing the gap size, as both lead to a stronger flow rarefaction.

\subsection{Effects of the gap size}

Geometry uncertainties due to manufacturing precisions are commonly encountered in microdevices. The real gap size falls in the range of $g_{0} \pm \Delta_{g}$, where $g_{0}$ is the nominal gap size reported based on measurements, and $\Delta_{g}$ is the combined error from measuring tools and methods. In examples, the nominal gap sizes are 1.4 and $1.6 \mu \mathrm{m}$, and $\Delta_{g}$ is chosen to be $0.25 \mu \mathrm{m}$, which is common for real cases. Two Knudsen numbers have been considered, which are 0.003 for slip 


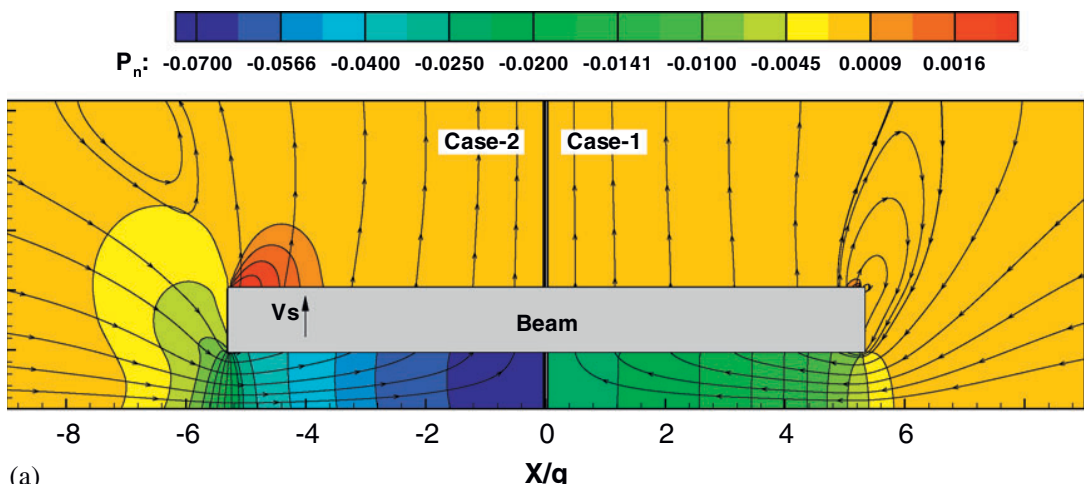

(a)

$\mathrm{X} / \mathrm{g}$

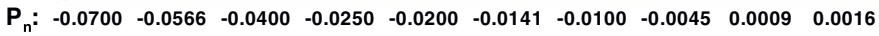

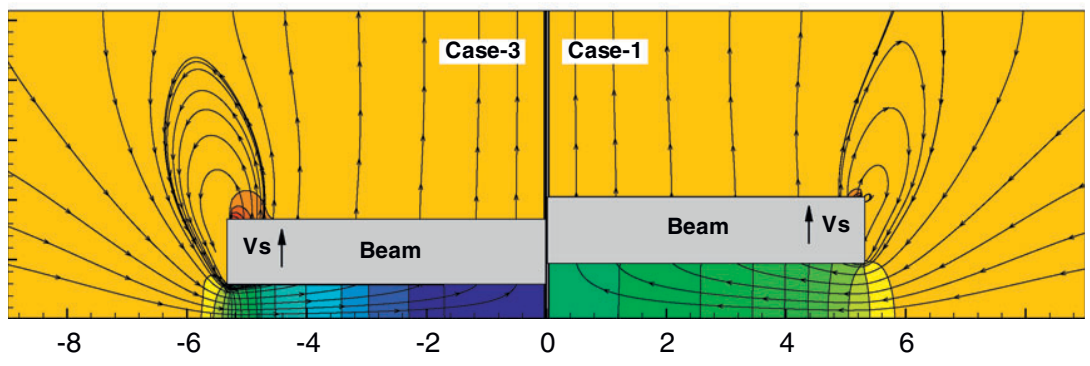

(b)

$\mathbf{X} / \mathbf{g}$

Figure 5. Pressure fields and streamlines with different rarefaction conditions and geometry. The reference length is chosen to be the gap size in case- 1 , and the reference pressure is chosen to be the ambient pressure for each case. The $x$-axis and the $y$-axis are dependent. $P_{n}=P_{22} / P_{A}-1$ : (a) Different pressure and (b) different geometry.

Table V. Flow field and geometry conditions.

\begin{tabular}{lcc}
\hline Case & $x_{1}$ & $x_{2}$ \\
\hline case-1 & 10.56 & $5.839 \mathrm{e}-3$ \\
case-2 & 10.56 & 2.237 \\
case-3 & 17.44 & $5.839 \mathrm{e}-3$ \\
\hline
\end{tabular}

and 3 for free-molecular flows. Since the reference length in our Knudsen number definition is chosen to be the beam width, the range scopes for slip, transitional and free-molecular flows are, therefore, shifted down by an order of magnitude.

Assuming a normal distribution for the gap size, corresponding output PDFs for the damping coefficient at different Knudsen numbers are obtained using probability transformation based on the compact model. Results are shown in Figure 6 with geometry conditions for each case summarized in Table VI. 


\section{GUO ET AL.}
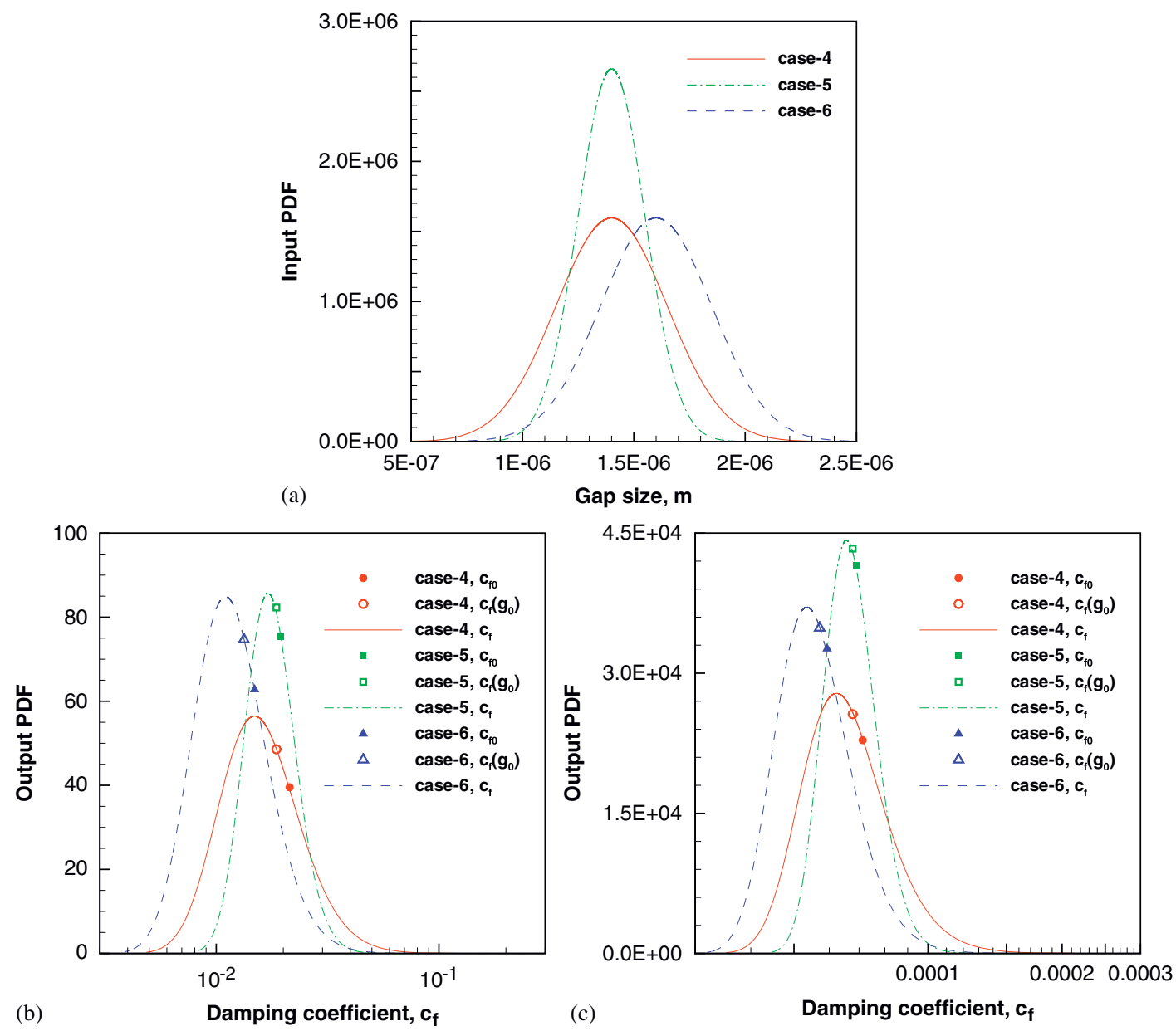

Figure 6. Probability density function with respect to uncertainties of the gap size: (a) input PDF of $g$; (b) output PDF of $c_{f}$ at $K n^{(b)}=0.003$; and (c) output PDF of $c_{f}$ at $K n^{(b)}=3$.

Table VI. Uncertainty conditions for input gas sizes, $b=18 \mu \mathrm{m}, t=1 \mu \mathrm{m}$.

\begin{tabular}{lccc}
\hline Case & $g_{0}, \mu \mathrm{m}$ & $\sigma_{g}, \mu \mathrm{m}$ & $x_{2}=K n^{(b)}$ \\
\hline case-4 & 1.4 & 0.25 & $0.003 ; 3$ \\
case-5 & 1.4 & 0.15 & $0.003 ; 3$ \\
case-6 & 1.6 & 0.25 & $0.003 ; 3$ \\
\hline
\end{tabular}

It is important to point out that the average damping coefficient, $c_{f 0}$, is different from the damping coefficient for the averaged (nominal) gap size, $c_{f}\left(g_{0}\right)$. As shown in Table VII, the difference can be as much as $12.7 \%$ for case- 3 with $K n^{(b)}=0.003$. The bias decreases as the Knudsen number increases. 


\section{UNCERTAINTY QUANTIFICATION MODELS}

Table VII. Uncertainty conditions for input gas sizes, $b=18 \mu \mathrm{m}, t=1 \mu \mathrm{m}, \Delta=\left|c_{f 0}-c_{f}\left(g_{0}\right)\right| / c_{f 0}$.

\begin{tabular}{llc}
\hline Case & $K n^{(b)}$ & $\Delta,(\%)$ \\
\hline case-4 & 0.003 & 12.7 \\
& 3 & 5.02 \\
case-5 & 0.003 & 4.51 \\
& 3 & 1.72 \\
case-6 & 0.003 & 10.84 \\
& 3 & 3.77 \\
\hline
\end{tabular}
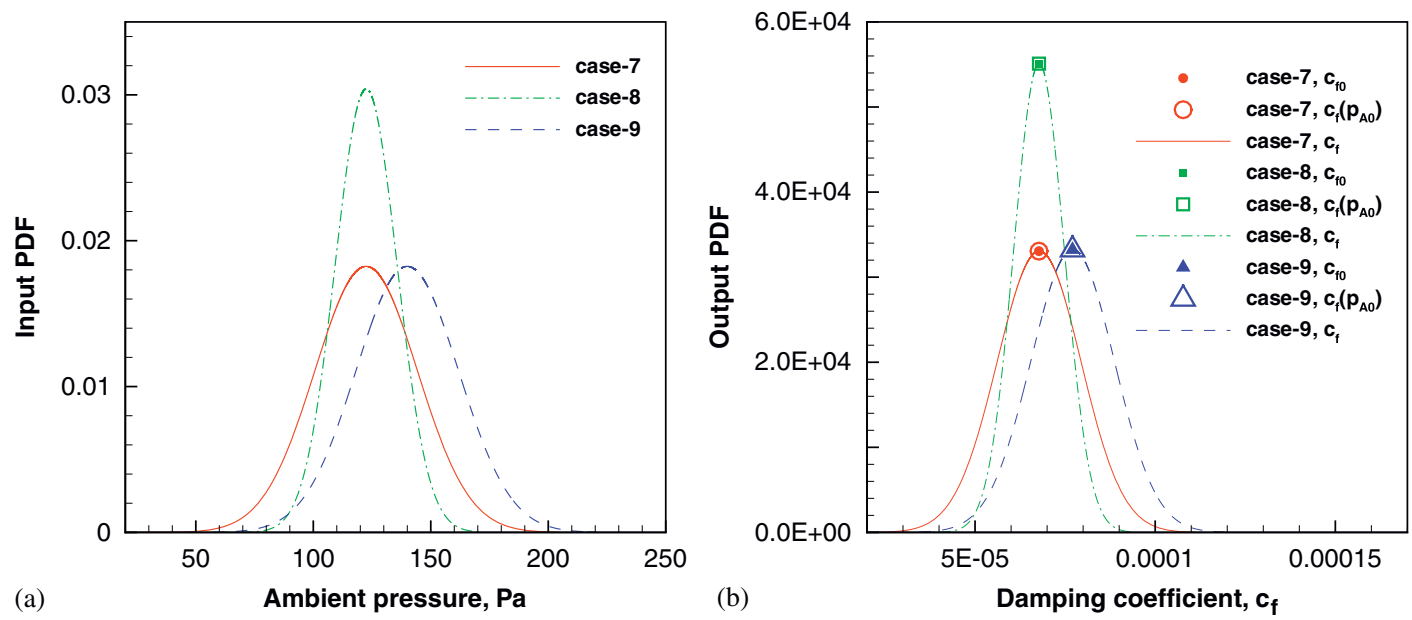

Figure 7. Probability density function with respect to uncertainties of the ambient pressure: (a) input PDF of $g$ and (b) output PDF of $c_{f}$ at $K n^{(b)}=3$.

Table VIII. Uncertainty conditions for input ambient pressures, $b=18 \mu \mathrm{m}, t=1 \mu \mathrm{m}$.

\begin{tabular}{lccc}
\hline Case & $x_{2}\left(p_{0}\right)$ & $\sigma_{p}\left(g / p_{0}\right)$ & $g, \mu \mathrm{m}$ \\
\hline case-7 & 3 & 0.25 & 1.4 \\
case-8 & 3 & 0.15 & 1.4 \\
case-9 & 2.6 & 0.25 & 1.4 \\
\hline
\end{tabular}

\subsection{Effects of the ambient pressure}

Another important source of uncertainties is the ambient pressure, which may be either due to drift (or fluctuations) in environment, or, due to accuracy of the pressure sensor. A similar analysis has been undertaken for pressure uncertainty study as that for the gap size shown earlier. The input and output PDFs considering different input mean and standard deviation are shown in Figure 7(a) and (b). Case conditions are specified in Table VIII. 
Table IX. Uncertainty conditions for input ambient pressures, $b=18 \mu \mathrm{m}, t=1 \mu \mathrm{m}$, $\Delta=\left|c_{f 0}-c_{f}\left(p_{A 0}\right)\right| / c_{f 0}$.

\begin{tabular}{lcc}
\hline Case & $g, \mu \mathrm{m}$ & $\Delta,(\%)$ \\
\hline case-7 & 1.4 & 0.06 \\
case-8 & 1.4 & 0.021 \\
case-9 & 1.4 & 0.043 \\
\hline
\end{tabular}

Unlike the gap size uncertainty effects, where the skewness of output PDF's are observed clearly, the resulting output PDFs with respect to normal pressure distributions are almost symmetric. In addition, the differences between the averaged damping coefficient, $c_{f 0}$, and the damping coefficient for the averaged ambient pressure, $c_{f}\left(p_{A 0}\right)$, are negligible, as shown in Table IX.

\subsection{Nonlinearity and sensitivity}

The nonlinearity and sensitivity effects on gas damping are discussed in this section. The former focuses on damping characteristics with respect to gas flow regimes, and the latter focuses on parametric dependence of gas damping. In Figure 8, damping uncertainties attributed to the gap size and ambient pressure have been shown, respectively. In all cases, we assume that input distributions are bounded normal distributions. From Figure 8(a), it can be seen that the difference between the mean damping coefficient, $c_{f 0}$, and the damping coefficient corresponding to the mean gap, $c_{f}\left(g_{0}\right)$, are most significant in regime of moderate Knudsen numbers. The reason is that gas damping depends linearly on gap size for both high Knudsen numbers-when the flow is freemolecular-and for small Knudsen numbers when the flow is essentially in the continuum regime. In the transitional regime of moderate Knudsen numbers the damping coefficient has a nonlinear behavior as has been discussed in detail, for example, in Reference [20]. For linear regime when the Knudsen number based on the gap height is large or negligibly small, the difference between mean of the prediction and the prediction for the mean input becomes trivial. In contrast, the differences between $c_{f 0}$ and $c_{f}\left(p_{A 0}\right)$ are negligible for all cases where the gap size varies from 1 to $2 \mu \mathrm{m}$.

As the plot in semi-log scale suggests the damping coefficient grows almost exponentially as the gap size decreases. In comparison, the relation between damping coefficient and the ambient pressure is close to linear, which implies that the damping is more sensitive to the gap size than to the pressure. According to both the nonlinearity and the sensitivity, uncertainty quantification of varying gap size should receive more attention when a comparison between deterministic, singlepoint numerical simulations and experimental measurements is done in the presence of significant uncertainty in input parameters.

\section{CONCLUSIONS}

The work focuses on uncertainty quantification of micro-scale gas damping in application to analysis of micro-electro-mechanical systems (MEMS). Both the general polynomial chaos expansion model and the compact squeeze-film damping (SFD) model based on rarefied flow simulations are proposed. Validation and verification have been carried out in terms of comparison with the experimental data, statistical tests and probability distribution analysis. A good agreement has been 


\section{UNCERTAINTY QUANTIFICATION MODELS}

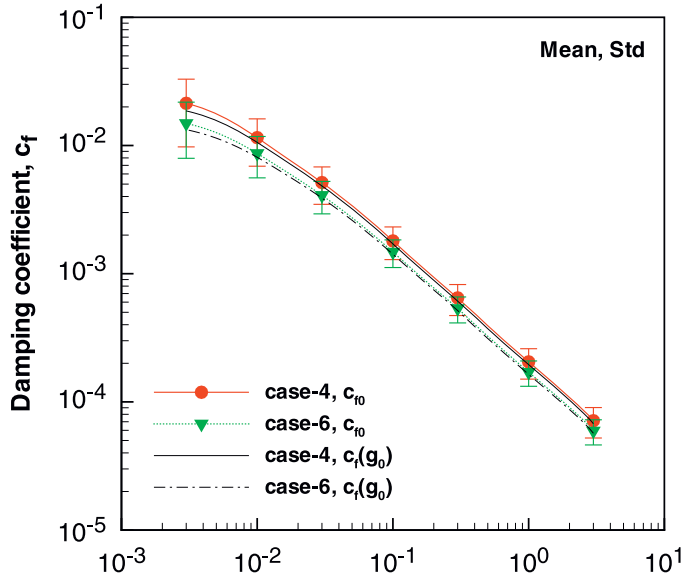

(a)

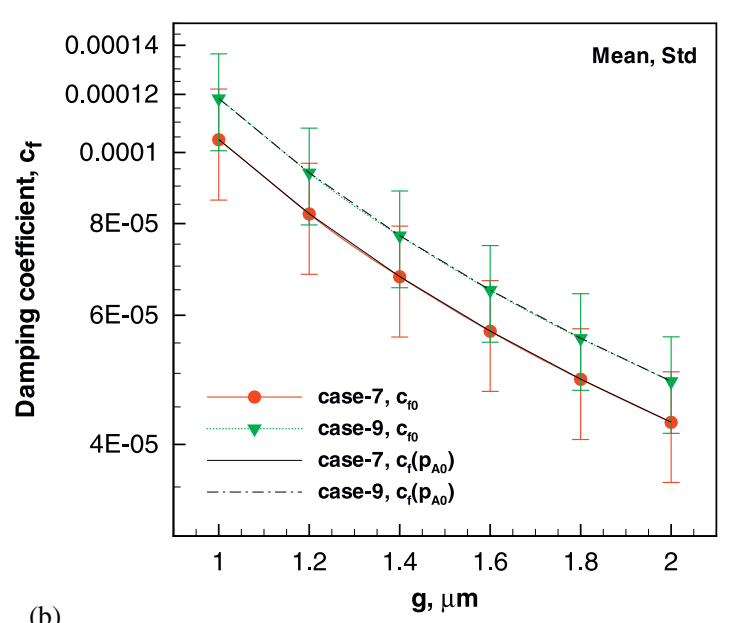

Figure 8. Effects of Nonlinearity: comparison between the average damping, $c_{f 0}$, and damping of the average input parameter, $c_{f}\left(x_{0}\right)$ : (a) Gap size and (b) ambient pressure.

obtained. In particular, the compact model turns out to be more convenient than the gPC model for damping uncertainty quantification discussed here. Based on the present work, damping is very sensitive to the gap size and the ambient pressure. In particular, damping coefficient grows almost exponentially as the gap size decreases. Additionally, the difference between the average damping coefficient and the damping coefficient for the average gap size cannot be ignored for cases in the transitional rarefied flow regime.

\section{ACKNOWLEDGEMENTS}

The work is supported by NNSA Center for Prediction of Reliability, Integrity and Survivability of Microsystems (PRISM) at Purdue University under contract DE-FC52-08NA28617.

\section{REFERENCES}

1. Zook J, Burns D, Guckel H, Sniegowski J, Engelstad R, Feng Z. Characteristics of polysilicon resonant microbeams. Sensors Actuators A: Physical 1992; 35(1):51-59.

2. Hosaka H, Itao K, Kuroda S. Damping characteristics of beam-shaped micro-oscillators. Sensors and Actuators A: Physical 1995; 49(1-2):87-95.

3. Veijola T. Compact models for squeezed-film dampers with inertial and rarefied gas effects. Journal of Micromechanics and Microengineering 2004; 14(7):1109-1118.

4. Gallis MA, Torczynski JR. An improved Reynolds-equation model for gas damping of microbeam motion. Journal of Microelectromechanical Systems 2004; 13(4):653-659.

5. Ozdoganlar OB, Hanshce BD, Carne TG. Experimental modal analysis for micro-electro-mechanical systems. Society for Experimental Mechanics 2005; 45(6):498-506.

6. Sumali H. Squeeze-film damping in the free molecular regime: model validation and measurement on a MEMS. Journal of Micromechanics and Microengineering 2007; 17(11):2231-2240.

7. Pan F, Kubby J, Peeters E, Tran AT, Mukherjee S. Squeeze film damping effect on the dynamic response of a MEMS torsion mirror. Journal of Micromechanics and Microengineering 1998; 8:200-208.

8. Chang KM, Lee SC, Li SH. Squeeze film damping effect on a MEMS torsion mirror. Journal of Micromechanics and Microengineering 2002; 12(5):556. 


\section{GUO ET AL.}

9. Bao M, Yang H. Squeeze film air damping in MEMS. Sensors and Actuators A: Physical 2007; 136(1):3-27.

10. Mohite SS, Sonti VR, Pratap R. A compact squeeze-film model including inertia, compressibility, and rarefaction effects for perforated 3-d MEMS structures. Journal of Microelectromechanical Systems 2008; 17(3):709-723.

11. Jin Y, Wang ZF, Lim PC, Pan DY, Wei J, Wong CK. MEMS vacuum packaging technology and applications. Electronics Packaging Technology, 2003 5th Conference (EPTC 2003), Singapore, 2003; 301-306.

12. Brown AS. MEMS across the valley of death. Mechanical Engineering 2006; 128:26-30.

13. Allen M, Raulli M, Maute K, Frangopol DM. Reliability-based analysis and design optimization of electrostatically actuated MEMS. Computers and Structures 2004; 82(13-14):1007-1020.

14. Liu R, Paden B, Turner K. MEMS resonators that are robust to process-induced feature width variations. Journal of Microelectromechanical Systems 2002; 11(5):505-511.

15. Agarwal N, Aluru NR. A stochastic Lagrangian approach for geometrical uncertainties in electrostatics. Journal of Computational Physics 2007; 226(1):156-179. DOI: 10.1016/j.jcp.2007.03.026.

16. Agarwal N, Aluru NR. Stochastic analysis of electrostatic MEMS subjected to parameter variations. Journal of Microelectromechanical Systems 2009; 18(6):1454-1468.

17. Bird GA. Molecular Gas Dynamics and the Direct Simulation of Gas Flows. Clarendon Press: Oxford, 2003.

18. Alexeenko AA, Gimelshein SF, Muntz EP, Ketsdever AD. Kinetic modeling of temperature driven flows in short microchannels. International Journal of Thermal Sciences 2006; 45(11):1045-1051.

19. Xiu D, Karniadakis GE. The Wiener-Askey polynomial chaos for stochastic differential equations. SIAM Journal on Scientific Computing 2002; 24(2):619-644.

20. Guo X, Alexeenko A. New compact model of squeeze-film damping based on rarefied flow simulations. Journal of Micromechanics and Microengineering 2009; 045026.

21. Ghanem RG, Spanos P. Stochastic Finite Elements: A Spectral Approach. Springer: Berlin, 1991.

22. Ghanem RG. Scales of fluctuation and the propagation of uncertainty in random porous media. Water Resources Research 1998; 34:2123.

23. Ghanem RG. Ingredients for a general purpose stochastic finite element formulation. Computer Methods in Applied Mechanics and Engineering 1999; 168:19-34.

24. Xiu D. Fast numerical methods for stochastic computations: a review. Communications in Computational Physics 2008; 5:242-272.

25. Xiu D, Hesthaven JS. High-order collocation methods for differential equations with random inputs. SISC 2005; 27(3): $1118-1139$.

26. Xiu D. Efficient collocational approach for parametric uncertainty analysis. Communications in Computational Physics 2007; 2(2):293-309.

27. Meirovitch L. Principles and Techniques of Vibrations (2nd edn). Prentice-Hall: Englewood Cliffs, NJ, 2000.

28. Mieussens L, Struchtrup H. Numerical comparison of Bhatnagar-Gross-Krook models with proper Prandtl number. Physics of Fluids 2004; 16(8):2797-2813.

29. Devore JL. Probability and Statistics for Engineering and the Sciences (6th edn). Brooks Cole: Belmont, CA, 2004. 